\title{
El desarrollo del paquete BiomSoft y sus aplicaciones en el análisis biomecánico del movimiento humano
}

\author{
K. GIANIKELLIS*, J.J PANTRIGO FERNÁNDEZ**, A. BOTE*; A. VARA GAZAPO* \\ * Facultad de Ciencias de la Actividad Física y del Deporte. Laboratorio de Biomecánica del Movimiento \\ Humano y de Ergonomía. Universidad de Extremadura. \\ ** Escuela Superior de Ciencias Experimentales y Tecnología. Universidad Rey Juan Carlos.
}

\begin{abstract}
Resumen
El análisis biomecánico del movimiento humano tiene aplicaciones, en el ámbito médico, ocupacional y deportivo analizando la técnica y diseñando material deportivo de altas prestaciones. El objetivo de este trabajo ha sido presentar el software desarrollado en el Laboratorio de Biomecánica del Movimiento Humano y de Ergonomía de la Facultad de Ciencias del Deporte de la Universidad de Extremadura que permite analizar el movimiento humano normal y patológico. El paquete BiomSoft desarrollado en entorno MATLAB 5.3 permite el tratamiento y la representación de los datos procedentes del análisis biomecánico aplicando diferentes técnicas de suavizado e interpolación de los datos cinemáticos y cálculo de las magnitudes cinemáticas lineales y angulares, la creación y uso de una base de datos de parámetros inerciales, análisis dinámico inverso y determinación de los momentos musculares netos y del trabajo mecánico realizado, análisis espectral de los datos Electromiográficos y de Estabilometría, representaciones gráficas y animaciones de modelos alámbricos, y simulación de procesos de tratamiento digital de señales (filtrado, padding, FFT, enventanado, etc.) con objetivos didácticos. Por tanto, se ha desarrollado un software que permite la descripción, análisis y evaluación de la motricidad humana en diferentes ámbitos de la Biomecanica del Movimiento Humano, además de ser muy útil para la enseñanza y las prácticas de laboratorio.
\end{abstract}

Palabras claves: análisis biomecánico, movimiento humano, tratamiento de datos, software.

\section{Summary}

The package BiomSoft is a set of MATLAB functions blocked in modules, useful and user friendly which allow the parameterisation of general motor patterns. BiomSoft enables to treat digital signal in different ways, calculate kinematic and kinetic magnitudes, provide different anthropometric models and present results graphically, which is useful in teaching. The objective of BiomSoft is to provide a complete processing and analysing Biomechanics data system with the possibility of to incorporate new functions and to perform particular applications in sports, medical and occupational Biomechanics, as well as in teaching and learning.

Key Words: biomechanics, human locomotion, signal processing, software.

Correspondencia:

Konstantinos Gianikellis,

Facultad de Ciencias del Deporte,

Avda. de la Universidad s/n. Cáceres.

E-mail:kgiannik@unex.es 


\section{Introducción}

La Biomecánica del Aparato locomotor puede definirse como conjunto de conocimientos interdisciplinares generados a partir de utilizar, con el apoyo de otras ciencias biomédicas, los conocimientos de la mecánica y distintas tecnologías en, primero, el estudio del comportamiento humano, $y$, segundo, resolver los problemas que le provocan las distintas condiciones a las que puede verse sometido. Hoy en día, la Biomecánica se desarrolla en los ámbitos: i) - deportivo, analizando y evaluando la práctica deportiva para mejorar el rendimiento y prevenir las lesiones, desarrollar técnicas de entrenamiento y diseñar complementos deportivos de altas prestaciones, ii) - médico, analizando las patologías que aquejan al cuerpo humano para generar soluciones capaces de evaluarlas, repararlas y paliarlas, y iii) - ocupacional, analizando la relación mecánica que el cuerpo humano sostiene con los elementos con los que interactúa en distintos ambientes - laboral, docente, doméstico o de ocio - para adaptarlos a sus necesidades y características (Vera y otros, 1992¹).

La metodología de investigación de la Biomecánica del Movimiento Humano (Fig. 1) esta orientada en conocer, las solicitaciones mecánicas a las que se ven sometidos los tejidos y elementos integrados en los complejos articulares del sistema biológico en movimiento y evaluar la calidad de los procesos de control (Cappozo, 19912; Bartlett, $1992^{3}$ ) tarea que no es siempre fácil, debido, primero, a sus características y propiedades mecánicas, y, segundo, a limitaciones metodológicas e instrumentales. En este sentido, es necesario com- binar la información obtenida respecto a, a) la geometría y la cinemática del movimiento, una vez conocidas las coordenadas tridimensionales de los marcadores anatómicos, mediante el uso de técnicas de fotogrametría tridimensional, b) las fuerzas externas ejercidas sobre la superficie de apoyo mediante el uso de plataforma de fuerzas, y, c) los datos antropométricos, obtenidos midiendo directamente sobre los sujetos del estudio o utilizando modelos antropométricos.

El procesamiento de estos datos se basa en las leyes de la mecánica utilizando el modelo de los segmentos rígidos articulados. La parametrización de la información obtenida permite describir, analizar y evaluar objetivamente los patrones motores en términos de eficacia biomecánica, puesto que, el sistema biológico tiene sus propias características, especialmente a lo que se refiere a la intervención muscular. Para dar respuesta a las necesidades del análisis biomecánico del movimiento humano desde hace dos décadas se están desarrollando diferentes sistemas de registro y análisis, al principio en dos y después en tres dimensiones. Sin embargo, nos encontramos con el problema de que so herramientas cerradas e incompletas, y que en su mayoría no permiten parametrizar adecuadamente el patrón motor bajo estudio, en el dominio del tiempo y de la frecuencia, obteniendo toda la información que requiere su evaluación. Otro inconveniente con los sistemas comercializados son las limitaciones respecto a la representación gráfica de los parámetros de interés y el tratamiento estadístico de los resultados sin necesidad de exportar los datos.

El objetivo del trabajo que se presenta a conti-

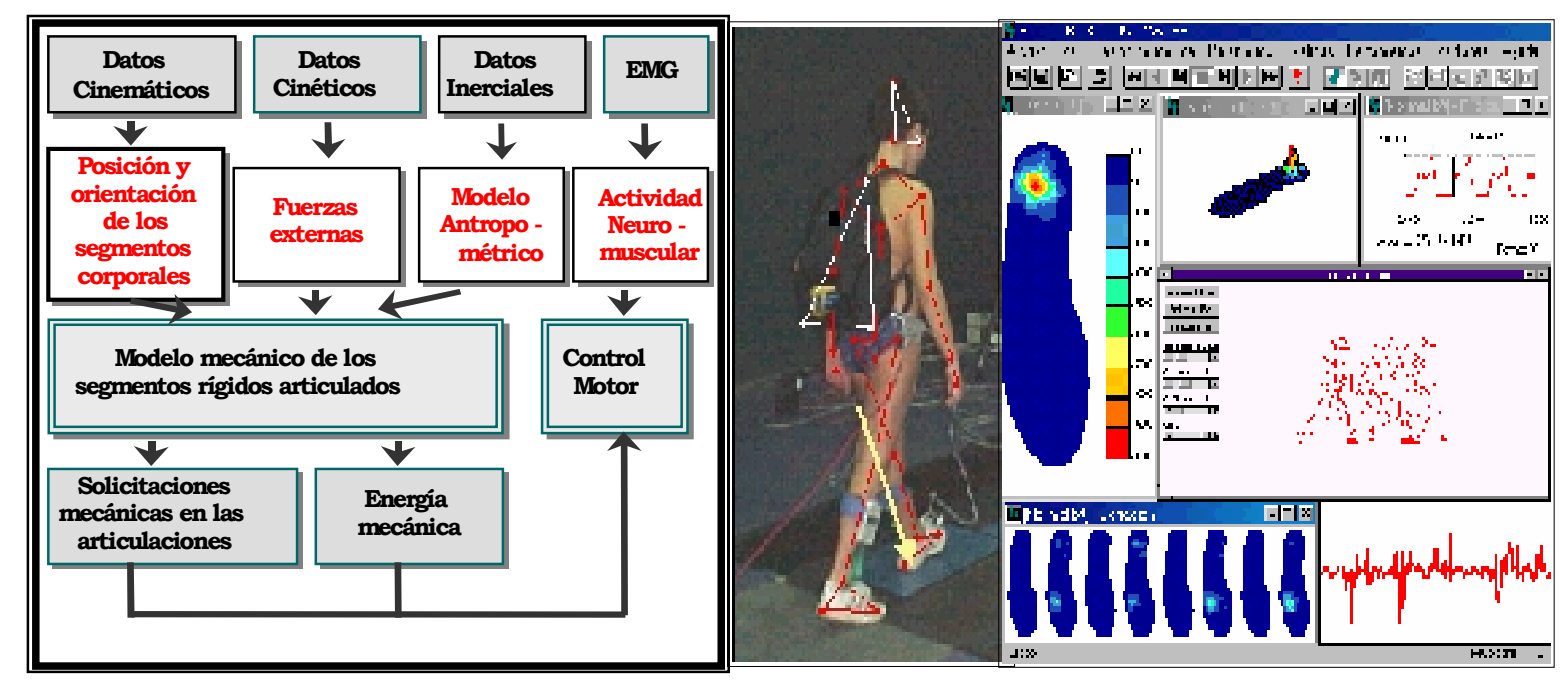

Figura .1 
nuación ha sido desarrollar, en primer lugar, en entorno MATLAB (The MATHWORKS, 19974, $1999^{5}$ ), el «BiomSoft», como un programa general de análisis biomecánico del movimiento humano que permita integrar la información cinemática, cinética y antropométrica, y, en segundo lugar, llevar a cabo aplicaciones específicas para analizar los patrones motores en el ámbito deportivo, aplicaciones dirigidas a analizar la motricidad humana en el ámbito de la discapacidad física, y aplicaciones orientadas a la adaptación de las condiciones del entorno laboral para mejorar la productividad y prevenir lesiones músculo - esqueléticas causadas por el nivel del esfuerzo físico, la repetitividad de las acciones motrices y el mantenimiento prolongado de posturas forzadas.

\section{Material y métodos}

El paquete BiomSoft fue desarrollado para el sistema operativo Windows de Microsoft en MATLAB 5.3, aceptando como entrada archivos de datos ASCII exportados desde los diferentes sistemas electrónicos y equipos de medida del laboratorio de Biomecánica del Movimiento Humano y de Ergonomía, como son el sistema de fotogrametría - vídeo 3D (KINESCAN), el sistema de plataformas de fuerzas (DINASCAN) y un sistema de EMG/ELG desarrollado en el mismo Laboratorio, y, los parámetros inerciales de una serie de mode- los antropométricos que están en una base de datos, según el diagrama de flujo que aparece a continuación (Fig. 2). Como se puede observar el programa incluye los siguientes módulos:

- la parte del análisis cinemático

- la parte del análisis cinético

- la base de datos de los parámetros inerciales la toolbox de tratamiento digital, suavizado e interpolación de los datos posición - tiempo

- la parte del análisis estabilométrico

- la parte de las representaciones gráficas y animaciones de los modelos alámbricos

\section{Resultados y discusión}

El BiomSoft (Fig. 3) permite::

- Filtrar e interpolar los datos posición-tiempo con funciones Spline utilizando el algoritmo Generalised Cross-Validatory Splines desarrollado por Woltring , $1986^{6}$, Challis and Kerwin, $1988^{7}$, Vaughan, $1982^{8}$.

- Calcular todos los parámetros cinemáticos lineales y angulares. Eje: permite calcular el vector orientación y los ángulos de Cardan - Euler (Spoor and Veldpaus, $1980^{\circ}$, Woltring, $1991^{10}$, Gianikellisn et al. $\left.1998^{11}\right)$. Esta posibilidad es de gran importancia para evaluar la postura y la estabilidad postural en función de ángulos relativamente pequeños menores de $10^{\circ}$ (Fig. 3 ).

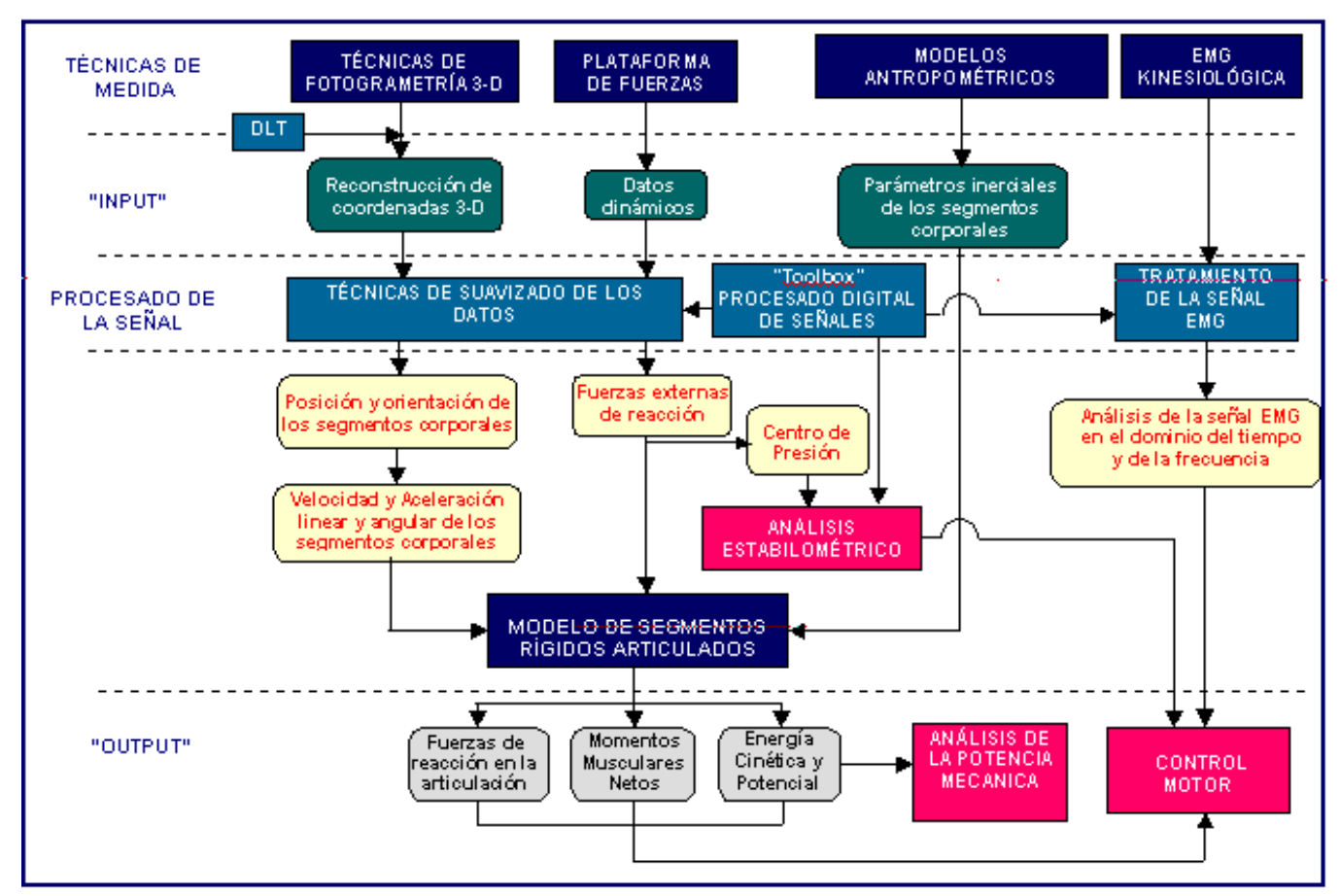

Figura 2 


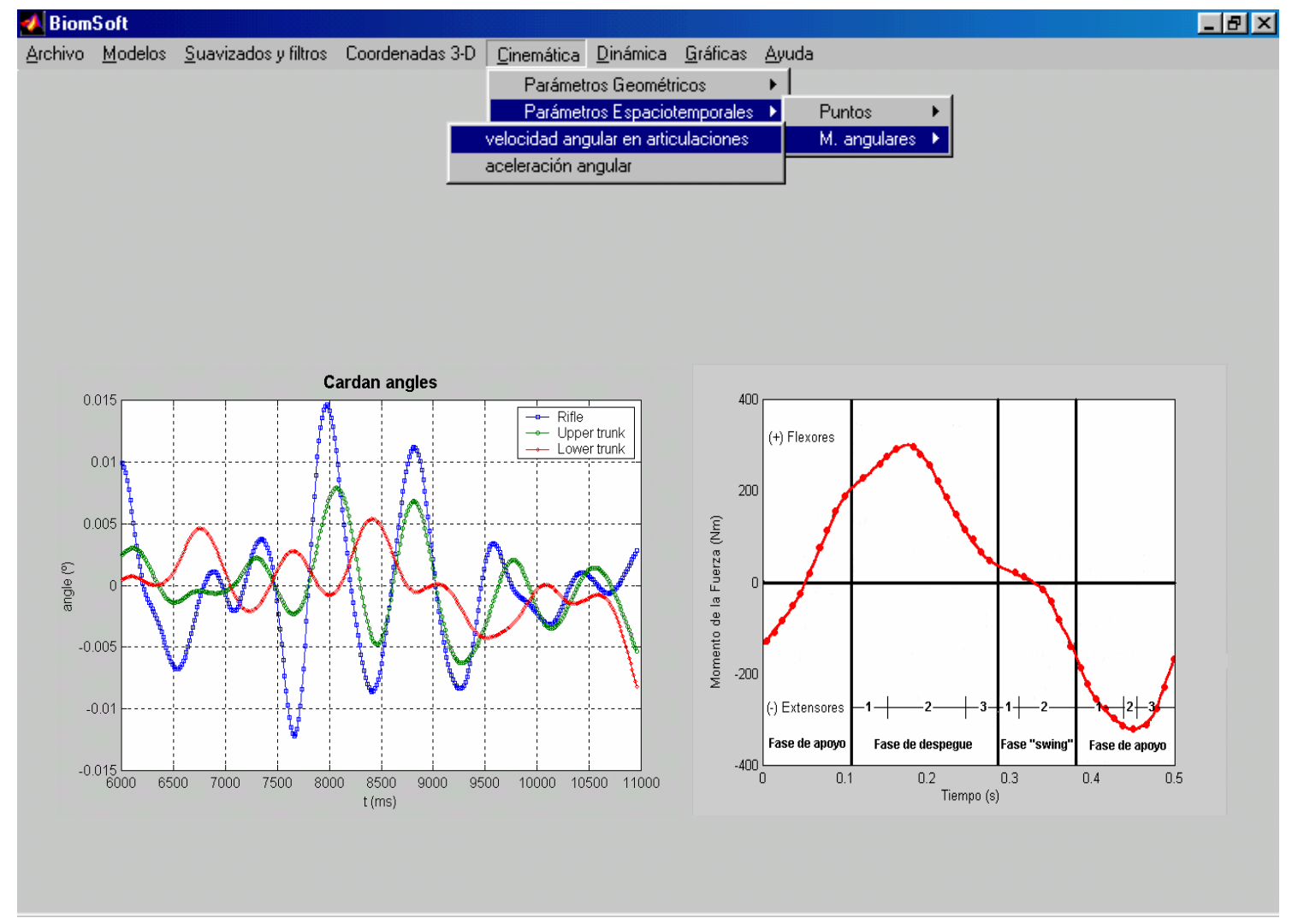

Figura 3

- Calcular el nivel de las fuerzas de reacción y los momentos musculares netos en las articulaciones, así como, la cantidad de energía mecánica que se transfiere de un segmento a otro y el nivel de potencia mecánica que se genera o se absorbe en las unidades biocinemáticas del aparato locomotor (Dainty and Norman, 1987'12) (Fig. 3). Esta infor- mación es fundamental para la planificación del entrenamiento deportivo especialmente para la especificidad del entrenamiento.

- Permite obtener los parámetros antropométricos sobre el sujeto analizado por medio de fotogrametría video tridimensional o hacer uso de diferentes modelos antropométricos (Hatze,
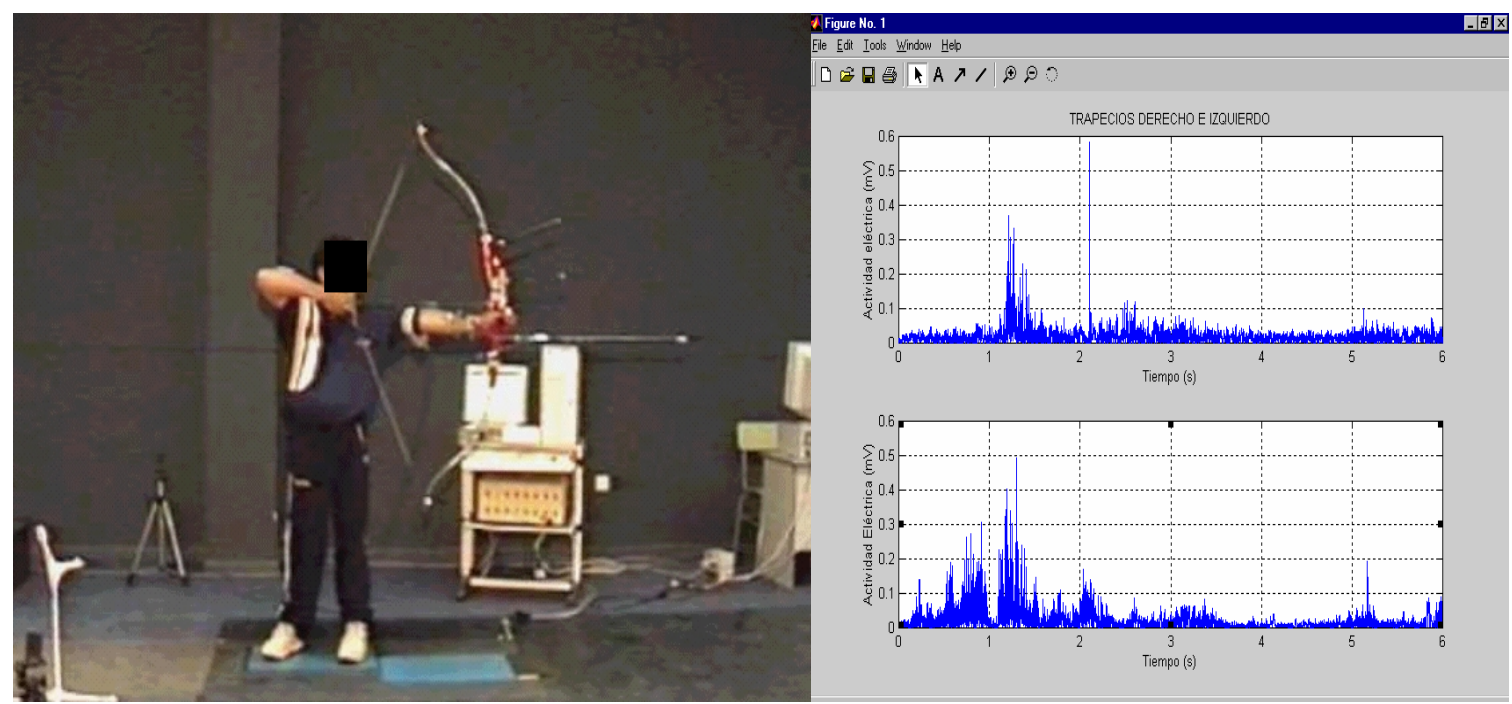

Figura 4 


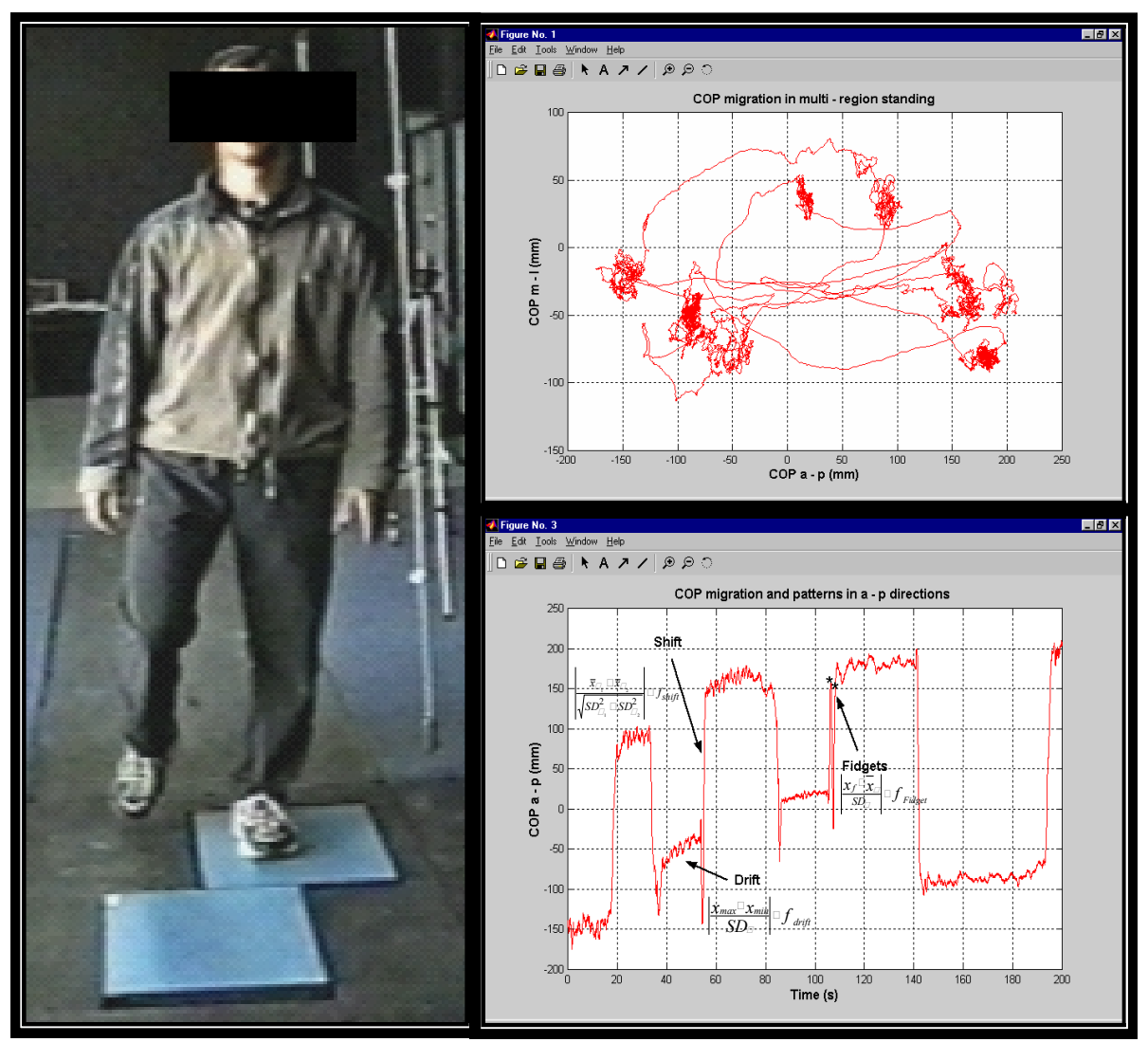

Figura 5

Zatsiorsky-Seluyanov, De Leva, Hanavan, Yeadon, Vaughan, Clauser, etc.)

- Analizar el EMG en el dominio del tiempo y de la frecuencia y detectar la fatiga muscular local (Fig. 4) (Gianikellis y otros, $1997^{13}$ )

- Analizar el estabilograma en el dominio del tiempo y de la frecuencia y detectar los problemas de control de la postura erguida especialmente en el contexto de la parálisis cerebral (Fig. 5) (Gianikellis y Maynar, 1998 ${ }^{14}$ ).
- Almacenar todos los parámetros temporales (instantes, fases, frecuencias, etc.) para poder ser utilizados en análisis posteriores.

- Representaciones gráficas y animaciones. (Fig. 6)

- Desarrollo de aplicaciones en los ámbitos deportivo, medico y ocupacional, permitiendo la posibilidad de realizar una simulación de los procesos de tratamiento digital de señales (filtrado, padding, FFT,

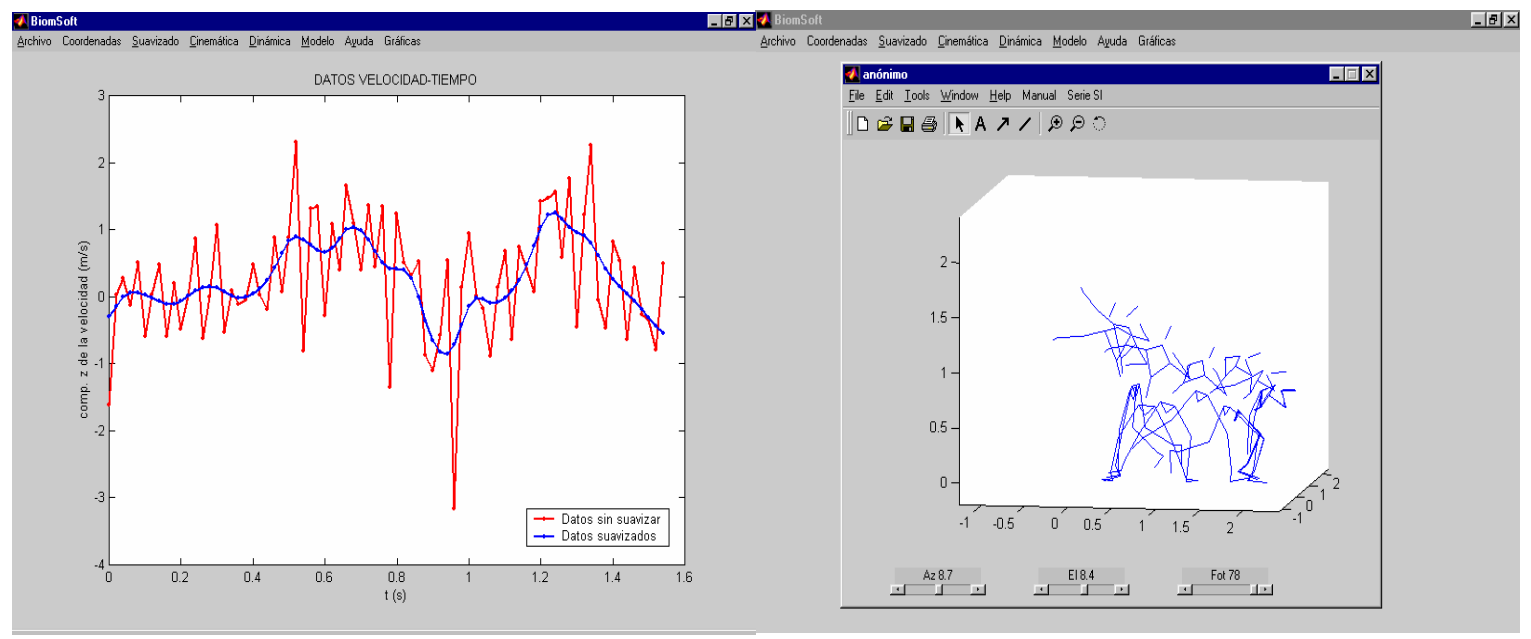

Figura 6 
enventanado, etc.) con objetivos didácticos (Fig.6).

\section{Conclusiones}

El paquete BiomSoft permite la descripción, análisis y evaluación de la motricidad humana en diferentes ámbitos de la Biomecánica del Movimiento Humano, además de ser muy útil para la enseñanza y las prácticas de laboratorio.

\section{Agradecimientos}

Este estudio ha sido parte del proyecto de investigación: Desarrollo de una línea de investigación aplicada a la evaluación, mediante análisis biomecánico de la motricidad de personas discapacitadas en actividades de la vida diaria y deportiva. Financiado por la Junta de Extremadura.

\section{Bibliografía}

1. Vera, P. y otros. Introducción a la biomecánica de los sistemas Biológicos, interrogantes y perspectivas en la Biomecánica del Aparato Locomotor. 1. El método Ingenieril aplicado al estudio de los sistemas Biológicos. Rev. S. And. Traum. y Ort. 1981, 2: $165-172$.

2. Cappozo, A.. Three-dimensional analysis of human walking: Experimental methods and associated artefacts. Human Movement Science, 1991, 10: 589 -602 .

3. Bartlett, R.M.. Biomechanical Analysis of Performance in Sport. Leeds: British Association of Sports Sciences, 1991.

4. The MATHWORKS Inc. Using MATLAB. Versión 5, 1999, (revisada para MATLAB 5.3).
5. The MATHWORKS Inc., Building GUIs with MATLAB. Versión 5, 1997, (revised for MATLAB 5.1).

6. Woltring, H. J. Fortran package for Generalized, Cross - Validatory spline smoothing and differentiation. Advances in Engineering Software, 1986, 8(2): 104-113.

7. Challis, J.H.; Kerwin, D.G.. An evaluation of splines in biomechanical data analysis. In Biomechanics XI - B (edited by G. De Groot, A.P.Hollander, P.A. Huijing and G.J. van Ingen Schenau), 1988, $1057-$ 1061. Amsterdam: Free University Press.

8. Vaughan, C.L.. Smoothing and differentiating of displacement data: an application of splines and digital filtering. International Journal of Bio-Medical Computing, 1982, 13:375-385.

9. Spoor, C.W.; Veldpaus, F.E.. Rigid body motion calculated from spatial co-ordinates of markers. Journal of Biomechanics, 1980, 13:391 - 393.

10. Woltring, H.J.. Representation and calculation of 3-D joint movement. Human Movement Science, 1991, 10:603-616.

11. Gianikellis, K.; Maynar, M. Fundamentos biomecánicos de la estabilometría y su aplicación en diferentes modalidades de los deportes de precisión. Biomecánica, 1998, VI (10): 37 - 44.

12. Dainty, D.A.; Norman, R.W. (eds). Standardising Biomechanical Testing in Sport. Champaign: Human Kinetics, 1987.

13. Gianikellis, K.; Maynar, M.; Arribas, F. La Electromiografia (EMG) como método para determinar la intervención muscular en los Deportes de Precisión. Icd, Investigación en Ciencias del Deporte, Consejo Superior de Deportes, 1997, 13: 107 $-121$.

14. Gianikellis K.; Maynar M; Durá J. V. A mechanical model for measuring in three dimensions the small amplitude coupled motion that characterizes motor patterns in shooting activities. ISBS' 98,1998 